\title{
BMI Trends, Socioeconomic Status, and the Choice of Dataset
}

\author{
Michael Grabner \\ HealthCore, Inc., Wilmington, DE, USA
}

\section{Key Words}

Body mass index $\cdot$ BMI $\cdot$ Obesity $\cdot$ Socioeconomic status $\cdot$ Health surveys $\cdot$ Ethnic differences

\begin{abstract}
Objective: This study is a descriptive investigation of trends in BMI in the USA over time, across race/ethnicity, gender, and socioeconomic status (SES) groups, and across different datasets. Methods: The study analyzes micro-level data from three widely used cross-sectional US health datasets: the National Health and Nutrition Examination Survey (NHANES), the National Health Interview Survey (NHIS), and the Behavioral Risk Factor Surveillance System (BRFSS), from the 1970s to 2008. Consistent race/ethnicity and SES groups are constructed for all datasets. SES is measured by education and income. Focusing on adults aged 20-74 years, the study estimates BMI time trends, distributional shifts, and incremental associations (gradients) with SES. Results: SES-BMI gradients are consistently larger for women than for men, differ across race/ethnicity groups, and are similar across datasets. Trends in mean BMI are comparable across White, Black and Hispanic males, while Hispanic females range between White and Black females. Self-reported BMI in the NHANES differs markedly from self-reports in the NHIS and BRFSS. Conclusion: The NHANES, NHIS, and BRFSS provide similar evidence regarding BMI trends over time and across race/ethnicity, gender, and SES groups. Racial disparities in BMI remain after adjusting for SES and should be studied further.
\end{abstract}

Copyright (c) 2012 S. Karger GmbH, Freiburg

\section{Introduction}

Obesity remains a major public health concern. Obesity levels among US adults have increased dramatically over the past 30 years, from about $14 \%$ in 1970 to $34 \%$ in 2008 [1, 2]. By 2020 , over $70 \%$ of the population is expected to be overweight or obese [3, 4]. Although the USA is an outlier, other developed countries are experiencing similar changes [5]. Obesity 
increases the risk of coronary heart disease, diabetes, hypertension, and other diseases, and is a significant preventable cause of death with more than 200,000 associated deaths per year $[6,7]$. The direct medical costs of obesity were projected at USD 115-151 billion for $2010[4]$.

Obesity levels vary considerably by gender, race/ethnicity, age, and socioeconomic status (SES) [2, 8-10]. SES is usually captured by income, education, or occupation, or a combination of these. In developed countries there typically is a strong negative relationship (a negative gradient) between SES and obesity for women, while the relationship for men and children is inconsistent. Furthermore, in the USA the prevalence of obesity is much more pronounced in African-Americans and Mexican-Americans than non-Hispanic Whites [10]. It has also been documented $[3,10]$ that severe obesity has grown even faster - the prevalence of obesity (BMI $\geq 30 \mathrm{~kg} / \mathrm{m}^{2}$ ) doubled from 1986 to 2000 , while class 3 obesity rates (BMI $\geq 40 \mathrm{~kg} / \mathrm{m}^{2}$ ) have quadrupled.

Much of prior analysis of US obesity trends has been focused on either single years of observation, single population groups, or a single dataset (most commonly the National Health and Nutrition Examination Survey, NHANES). The NHANES is regarded as a reliable source of prevalence estimates since it contains exam-based measurements in addition to self-reported measurements, which are known to be biased [11]. However, the NHANES works with relatively small sample sizes and has been conducted annually only since 1999. This presents challenges for subgroup analysis and the examination of long-run time trends. The National Health Interview Survey (NHIS) and the Behavioral Risk Factor Surveillance System (BRFSS), two other national health datasets, offer much larger sample sizes and have been conducted annually since 1957 and 1984, respectively, but are constrained to selfreported measurements.

This study has two main objectives: First, it explores similarities and differences in BMI trends among US adults across the NHANES, NHIS, and BRFSS over a long time horizon (1971-2008). This provides a more detailed picture of recent BMI trends as well as evidence regarding the comparability of these datasets. Second, the study investigates and quantifies the incremental association between SES, as measured by education and income, and BMI across all three datasets. These gradients provide rough estimates of the health returns (measured as BMI improvements) to education and income. To account for potential heterogeneity across subgroups, all analysis is stratified by gender (male/female) and/or race/ ethnicity (White/Black/Hispanic). Taken together, this study provides new information on health disparities in BMI across all major sociodemographic subgroups in the USA, using several independent data sources and a long-run perspective.

\section{Material and Methods}

\section{Variables}

The outcome of interest in this study is the patient's BMI level (calculated as height/weight ${ }^{2} ; \mathrm{kg} / \mathrm{m}^{2}$ ), the most common measure of obesity. BMI is less accurate than laboratory measures because it ignores variations in the distribution of body fat and muscle mass [12]. It remains the most preferred measure in obesity research since it is easy and inexpensive to calculate, and the only readily available measure in most population-based datasets. Most analyses in this study focus on (mean) BMI in order to examine trends across the height/weight spectrum.

SES is determined using completed years of education and personal real income. Education and income were usually reported in brackets and transformed into continuous variables. Complete and consistent race/ethnicity recodes were created across the three datasets (non-Hispanic Whites, nonHispanic Blacks, Hispanics). More information on data preparation is contained in the online supplementary material (available at http://content.karger.com/ProdukteDB/produkte.asp?doi=337018). 


\section{Description of Datasets}

Data are drawn from repeated cross-sections of the NHANES, NHIS, and BRFSS. All observations are from the public-release files.

The NHANES is a nationally representative cross-sectional survey of the US civilian non-institutionalized population conducted by the National Center for Health Statistics (NCHS) [13]. The first series of interviews and measurements took place from 1971 to 1975 (NHANES1), the second from 1976 to 1980 (NHANES2), and a third from 1988 to 1994. Since 1999, the NHANES has been carried out continuously, with new data being released every 2 years. At the writing of this study the last available dataset was from 2005 to 2006, bringing the total number of available series to 7. Unique among similar national datasets, the NHANES combines interviews with physical examinations in mobile examination centers that travel to different counties every year. The NHANES over-samples persons 60 years of age and older, Blacks, and Hispanics. Starting with the NHANES2 there are two sources of body measurements - self-reports and clinical examinations (NHANES1 only includes clinical measurements). Clinically measured height and weight are reported in metric units, while self-reports use imperial units and were converted by the author to metric standards.

The NHIS is also a nationally representative cross-sectional survey of the US civilian non-institutionalized population conducted by the NCHS, in conjunction with the US Bureau of the Census [14]. It has been conducted continuously since 1957, with major design revisions every 10-15 years, the last one in 1997. Like the NHANES it over-samples Blacks and Hispanics. The NHIS is based on in-person household interviews and samples up to 87,500 people per year.

The Minnesota Population Center has released a harmonized set of data and documentation based on the public release files of the NHIS, starting in 1969, called the 'Integrated Health Interview Series' or IHIS [15]. Information on height, weight, and Hispanic ethnicity is available starting in 1976, and this was chosen as the base year for this study's use of the IHIS. The NHIS only provides self-reports of height and weight; before 1997, these self-reports also included proxy reports from other members of the household. The analysis used the BMI variable calculated by the IHIS, which is based on the publicly released height and weight files.

The BRFSS is another cross-sectional survey of civilian non-institutionalized US adults aged 18 years or older, but it differs from the NHANES and NHIS in several respects [16]: First, it is entirely conducted via (landline and cell) phone, based on random-digit dialing. Second, it is state-based; all 50 states are individually responsible for data collection while the questionnaire is centrally designed by the Centers for Disease Control and Prevention (CDC). Third, it is the youngest dataset of the three, having been established in 1984.

One adult is interviewed per household in the BRFSS. The documentation notes that certain subgroups of the population have lower telephone coverage (people living in the South; minorities; people with low SES) and that the provided sample weights may not fully adjust for this [17]. 15 states participated in the first round of interviews in 1984; since 1996 all 50 states and D.C. have been included. This study uses BRFSS data starting in 1987 when a sufficient number of states $(\mathrm{N}=33)$ had joined. Previous studies have shown that national estimates built from BRFSS data are usually similar to those obtained using truly national surveys such as the NHIS, while cautioning that identical results cannot be expected given the differences in population coverage and methodology [16]. BRFSS files provide self-reports of height and weight as well as, from 1987 onwards, a calculated BMI variable. This study uses the BMI variable contained in the BRFSS. The BRFSS does not collect proxy information.

\section{Inclusion/Exclusion Criteria}

The following sample adjustments were performed: i) All persons for whom race/ethnicity recodes were either missing or who did not belong to any of the three race/ethnicity groups were removed. ii) All persons less than 20 or more than 74 years of age were removed (NHANES1 and 2 did not sample people aged 75 or older, and CDC obesity guidelines define adults as those aged 20 years or greater [18]). iii) All persons with missing sample weights or BMI values, or with $\mathrm{BMI}<10$ or $\mathrm{BMI}>70 \mathrm{~kg} / \mathrm{m}^{2}$ were removed (this last step removed a handful of observations with outliers or miscodes). iv) Pregnant women were excluded from the analysis whenever that information was available (NHANES/BRFSS: all years; NHIS: 1997-2008). Application of these criteria led to reductions in observations of 54\% (NHANES), 52\% (NHIS), and $24 \%$ (BRFSS), relative to the full initial samples. Pooled final sample sizes are 49,965 for the NHANES; $1,537,380$ for the NHIS; and 3,210,678 for the BRFSS. 
Design and Statistical Analysis

This study explores BMI trends along several dimensions using a variety of approaches. The first section presents trends in mean BMI over time, stratified by gender, race/ethnicity, and SES. All graphs of time trends were age-adjusted to the 2000 projected US population using 11 age categories [19]. This section also examines shifts in the BMI distribution. It calculates BMI histograms in 1976 and 2006 by gender and race/ethnicity, using the NHIS due to its long time horizon and large sample size. Complementary analysis uses the 15th, 50th, and 85th percentiles of the BMI distribution to calculate average growth rates in the 85/50 and 50/15 differences, which measure changes in skewness of the BMI distribution over time. The first section concludes by discussing differences between self-reported and exambased measurements of BMI.

The second section estimates SES-BMI gradients, i.e. the incremental (marginal) effect of education and income on BMI. Education is measured in years of completed schooling; the marginal effect describes the impact of an additional year of schooling. Income is measured in thousands of constant year 2000 USD ; the marginal effect describes the impact of an increase in real personal income of USD 1,000.-. Gradients are estimated using ordinary least squares (OLS); additional covariates in the regression models include age, age squared, interactions between age/age squared and year, and year fixed effects. Since education and income both affect BMI, the section also calculates their relative importance by comparing the coefficients on schooling and income in regressions that only contain either schooling or income to those from regressions that contain both variables.

All three datasets used in this study represent complex survey data and this design was taken into account during the analysis by using sample weights and clustering standard errors by primary sampling unit (PSU). All analysis was performed in STATA10 [20].

\section{Results}

\section{BMI Time Trends}

\section{Overall BMI Trends}

Figure $1 \mathrm{a}$ and $\mathrm{b}$ describe the secular changes in BMI for the different datasets, stratified by gender. BMI measures have consistently increased over the whole period, and the trends are similar across all three surveys. Average BMI has increased from about $25.5 \mathrm{~kg} / \mathrm{m}^{2}$ in the late 1970 s to over $28.5 \mathrm{~kg} / \mathrm{m}^{2}$ in 2005 , while the fraction of the population classified as obese has increased from around $15 \%$ to roughly $35 \%$ (based on NHANES data). There is a considerable difference between measured BMI values from the NHANES on the one hand and self-reported BMI values from the NHIS and BRFSS on the other (self-reported measures from the NHANES lie somewhere in between; see below).

\section{BMI Trends Stratified by Gender and Ethnicity}

Table 1 and fig. 1c (for the NHIS) describe the rise in BMI across gender and race/ ethnicity groups. Disregarding sampling error, all measures have increased for all subgroups over the past 30 years. BMI growth was stronger for women than for men by about two percentage points in all three datasets. Growth rates were roughly similar across race/ ethnicity groups. Black women display by far the highest BMI, and White women the lowest (the average difference between these two groups is $2.5 \mathrm{BMI}$ units in the NHIS, 2.8 in the BRFSS and 3 in the NHANES). Hispanic women are roughly in the middle. Among males, prevalence rates are similar across race/ethnicity groups. These characteristics appear very stable over time and have been partially documented before (for the NHANES see [1, 2]; for the BRFSS see [21]; for basic results in the NHIS 1997 see [22]). 


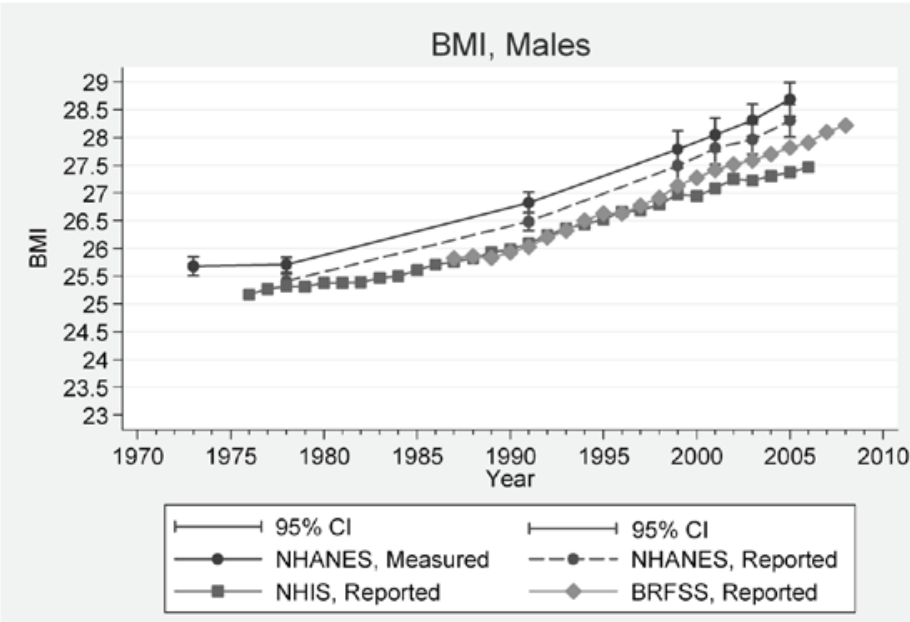

a

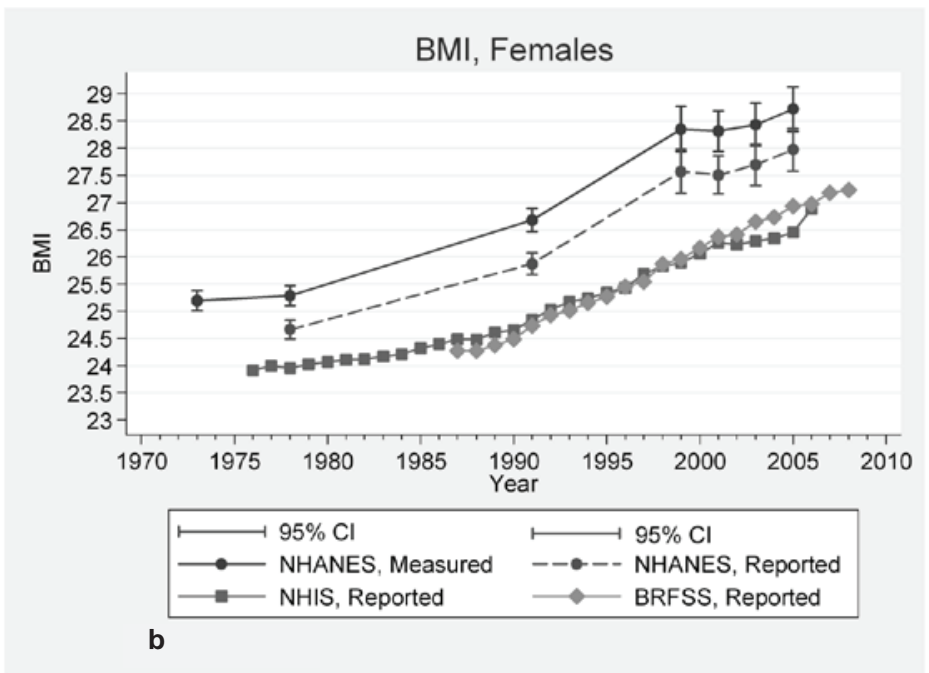

Fig. 1. Comparison of means across datasets and self-reports versus measurements. a BMI, males. b BMI, females. c BMI, stratified by gender and race/ ethnicity in the NHIS. Age 20-74 years. Weighted and age-adjusted

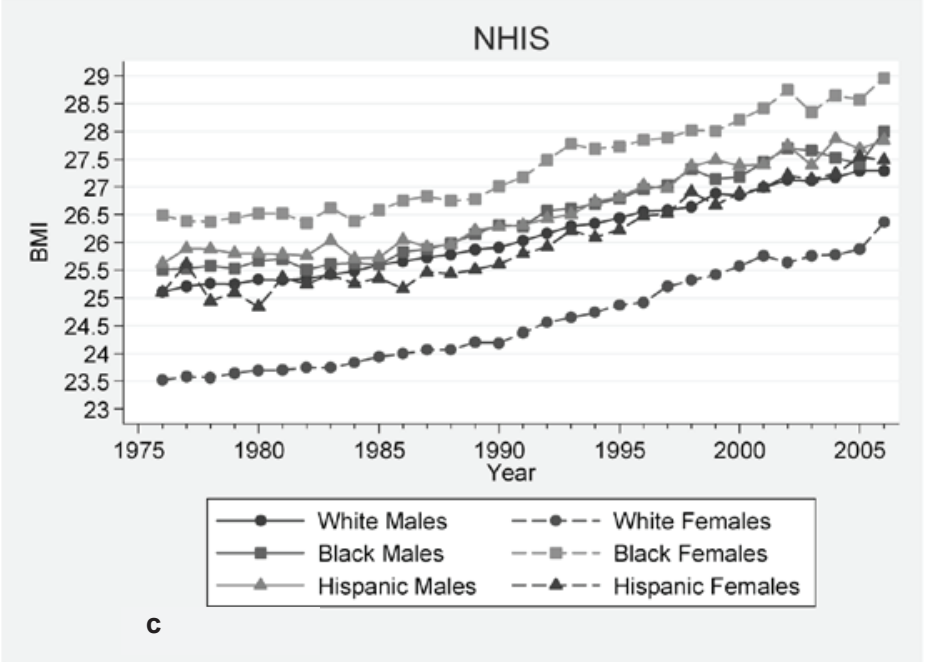
to the 2000 Census. 


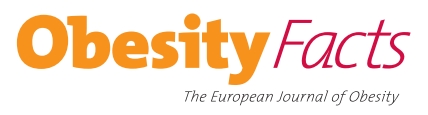

\begin{tabular}{l|l}
\hline Obes Facts 2012;5:112-126 \\
\hline $\begin{array}{l}\text { DOI: 10.1159/000337018 } \\
\text { Published online: March 2, } 2012\end{array}$ & $\begin{array}{l}\text { @ 2012 S. Karger GmbH, Freiburg } \\
\text { www.karger.com/ofa }\end{array}$ \\
\hline
\end{tabular}

Table 1. Trends in mean BMI ${ }^{\mathrm{a}}$

\begin{tabular}{|c|c|c|c|c|c|c|c|c|c|c|c|c|c|}
\hline & \multicolumn{5}{|c|}{ NHANES (exam-based) } & \multicolumn{5}{|l|}{ NHIS } & \multicolumn{3}{|l|}{ BRFSS } \\
\hline & $\begin{array}{l}\text { BMI } \\
1978\end{array}$ & $\begin{array}{l}\text { BMI } \\
1991\end{array}$ & $\begin{array}{l}\text { BMI } \\
2005\end{array}$ & $\begin{array}{l}1978- \\
2005 \\
\% \Delta\end{array}$ & $\begin{array}{l}1991- \\
2005 \\
\% \Delta\end{array}$ & $\begin{array}{l}\text { BMI } \\
1978\end{array}$ & $\begin{array}{l}\text { BMI } \\
1991\end{array}$ & $\begin{array}{l}\text { BMI } \\
2005\end{array}$ & $\begin{array}{l}1978- \\
2005 \\
\% \Delta\end{array}$ & $\begin{array}{l}1991- \\
2005 \\
\% \Delta\end{array}$ & $\begin{array}{l}\text { BMI } \\
1991\end{array}$ & $\begin{array}{l}\text { BMI } \\
2005\end{array}$ & $\begin{array}{l}1991- \\
2005 \\
\% \Delta\end{array}$ \\
\hline Total & 25.47 & 26.68 & 28.72 & 12.75 & 7.65 & 24.61 & 25.46 & 26.95 & 9.51 & 5.82 & 25.46 & 27.43 & 7.75 \\
\hline Males & 25.72 & 26.83 & 28.70 & 11.59 & 6.94 & 25.31 & 26.10 & 27.38 & 8.15 & 4.88 & 26.06 & 27.84 & 6.80 \\
\hline Females & 25.22 & 26.51 & 28.73 & 13.91 & 8.36 & 23.94 & 24.85 & 26.54 & 10.86 & 6.81 & 24.83 & 27.00 & 8.76 \\
\hline Whites & 25.28 & 26.41 & 28.36 & 12.21 & 7.40 & 24.40 & 25.22 & 26.64 & 9.16 & 5.62 & 25.26 & 27.10 & 7.28 \\
\hline Blacks & 26.86 & 28.00 & 30.48 & 13.48 & 8.85 & 25.99 & 26.87 & 28.17 & 8.40 & 4.84 & 26.86 & 29.03 & 8.09 \\
\hline Hispanics & 25.99 & 27.40 & 28.91 & 11.21 & 5.51 & 25.39 & 26.10 & 27.64 & 8.88 & 5.92 & 25.82 & 27.87 & 7.95 \\
\hline \multicolumn{14}{|l|}{ Education ${ }^{b}$} \\
\hline Low & 26.28 & 27.21 & 28.85 & 9.80 & 6.03 & 25.48 & 26.46 & 27.54 & 8.09 & 4.10 & 26.41 & 28.23 & 6.90 \\
\hline Medium & 25.43 & 27.16 & 29.50 & 15.98 & 8.60 & 24.45 & 25.63 & 27.34 & 11.81 & 6.66 & 25.73 & 28.04 & 8.98 \\
\hline High & 24.86 & 26.00 & 28.39 & 14.20 & 9.21 & 24.02 & 24.96 & 26.65 & 10.92 & 6.77 & 25.06 & 27.03 & 7.90 \\
\hline \multicolumn{14}{|l|}{ Income } \\
\hline Low & 26.02 & 27.37 & 29.19 & 12.17 & 6.63 & 25.28 & 26.29 & 27.62 & 9.25 & 5.04 & 26.23 & 28.32 & 7.96 \\
\hline Medium & 25.37 & 26.90 & 29.61 & 16.73 & 10.09 & 24.57 & 25.55 & 27.07 & 10.20 & 5.98 & 25.49 & 27.79 & 9.00 \\
\hline High & 24.92 & 25.98 & 27.93 & 12.08 & 7.48 & 24.04 & 24.87 & 26.58 & 10.59 & 6.89 & 25.01 & 26.81 & 7.18 \\
\hline
\end{tabular}

aAge 20-74 years. Weighted and age-adjusted to the 2000 Census.

bLow, medium and high education represents groups with $<12,=12$ and $>12$ years of completed education, respectively.

cLow, medium and high income represents groups with real personal income of $\leq$ USD 10,000.-, USD 10.000.- to 20,000.and $\geq$ USD 20,000.- in 2000 .

\section{BMI Distribution Shifts}

Figure $2 \mathrm{a}$ and $\mathrm{b}$ present histograms of BMI in the NHIS. The BMI distribution for men looks very similar for the three race/ethnicity groups, both in 1976 and 2006. Whites are consistently the least obese, and Blacks the most, with Hispanics in-between, but the differences are small. Over time, the variance increased modestly (average standard deviation = 3.8 in 1976 and 4.6 in 2006), and the fraction obese roughly tripled (Whites/Black/Hispanics: $8.3 \% / 12.3 \% / 11 \%$ in $1976 ; 25.4 \% / 29.5 \% / 26.5 \%$ in 2006 ).

Racial differences for women are more pronounced. There is a bigger difference between Whites and Hispanics, and between them and Blacks, whose obesity rate in 1976 was about twice as high as those of Whites and Hispanics. BMI variance has increased over time (average standard deviation $=4.9$ in 1976 and 5.9 in 2006), as did obesity prevalence (Whites/Black/Hispanics: 8.9\%/21.6\%/12.1\% in 1976; 24.5\%/38.9\%/27.2\% in 2006).

\section{BMI Distribution Skewness}

Looking at trends over time across percentiles of the BMI distribution provides additional evidence regarding distributional changes. Similar exercises in the NHANES [23, 24] and BRFSS $[25,26]$ have documented that the BMI distribution has both shifted to the right and become more right-skewed, explaining the disproportional growth in severe obesity noted earlier. This is also apparent in the NHIS. The average growth rate in the 85/50 gap (0.05 BMI units per year) exceeds growth in the 50/15 gap (0.02 BMI units) for White males. This difference is statistically significant $(\mathrm{p}<0.0001$ for a two-sided Wald test of parameter equality across the two linear growth equations). Black males (growth in the 85/50 BMI percentile gap $=0.042$ units per year; $50 / 15$ gap $=0.025 ; \mathrm{p}=0.0079)$, Hispanic males $(0.028 / 0.015 ; \mathrm{p}=0.0197)$, and White females $(0.064 / 0.044 ; \mathrm{p}<0.0001)$ also experienced 

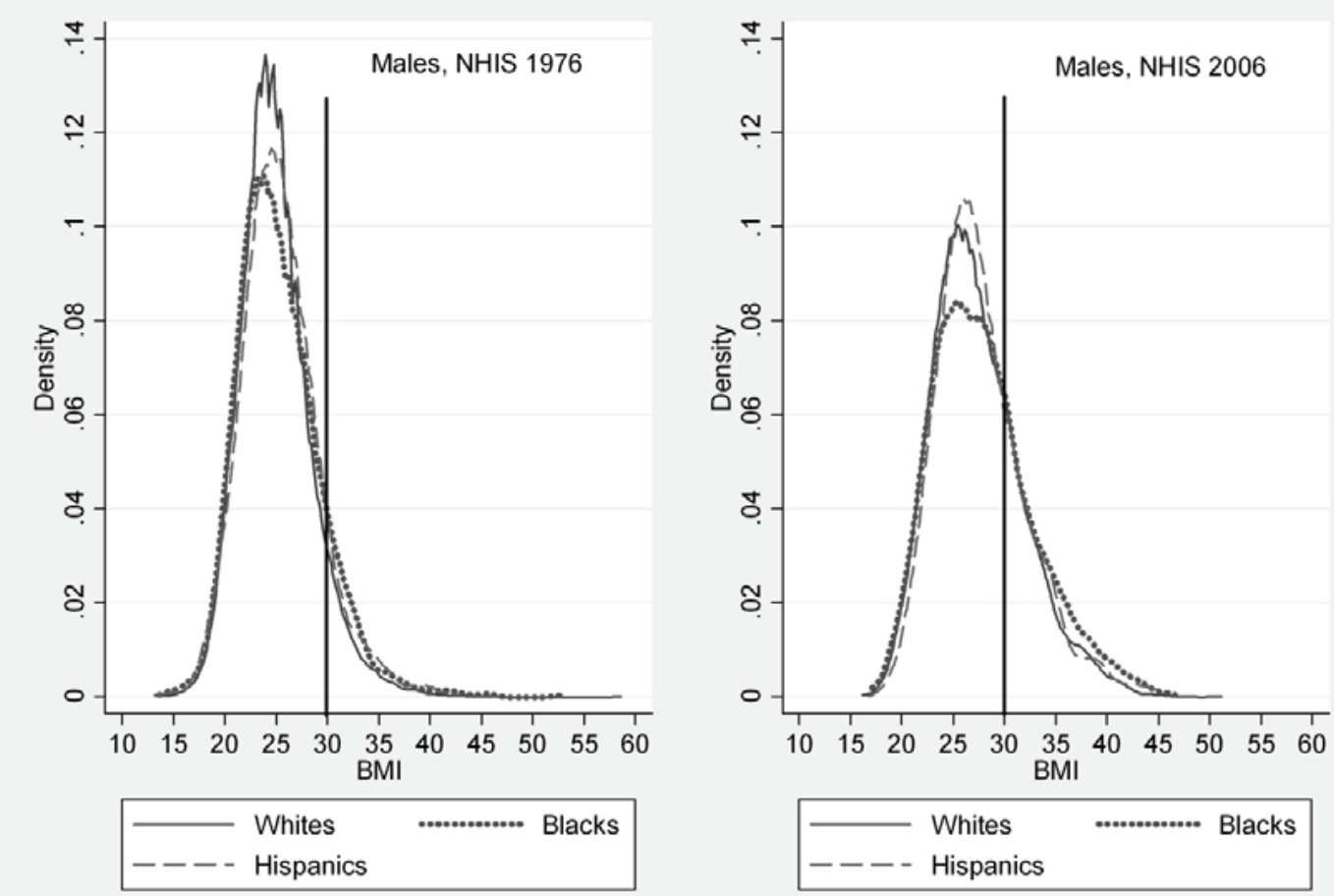

a
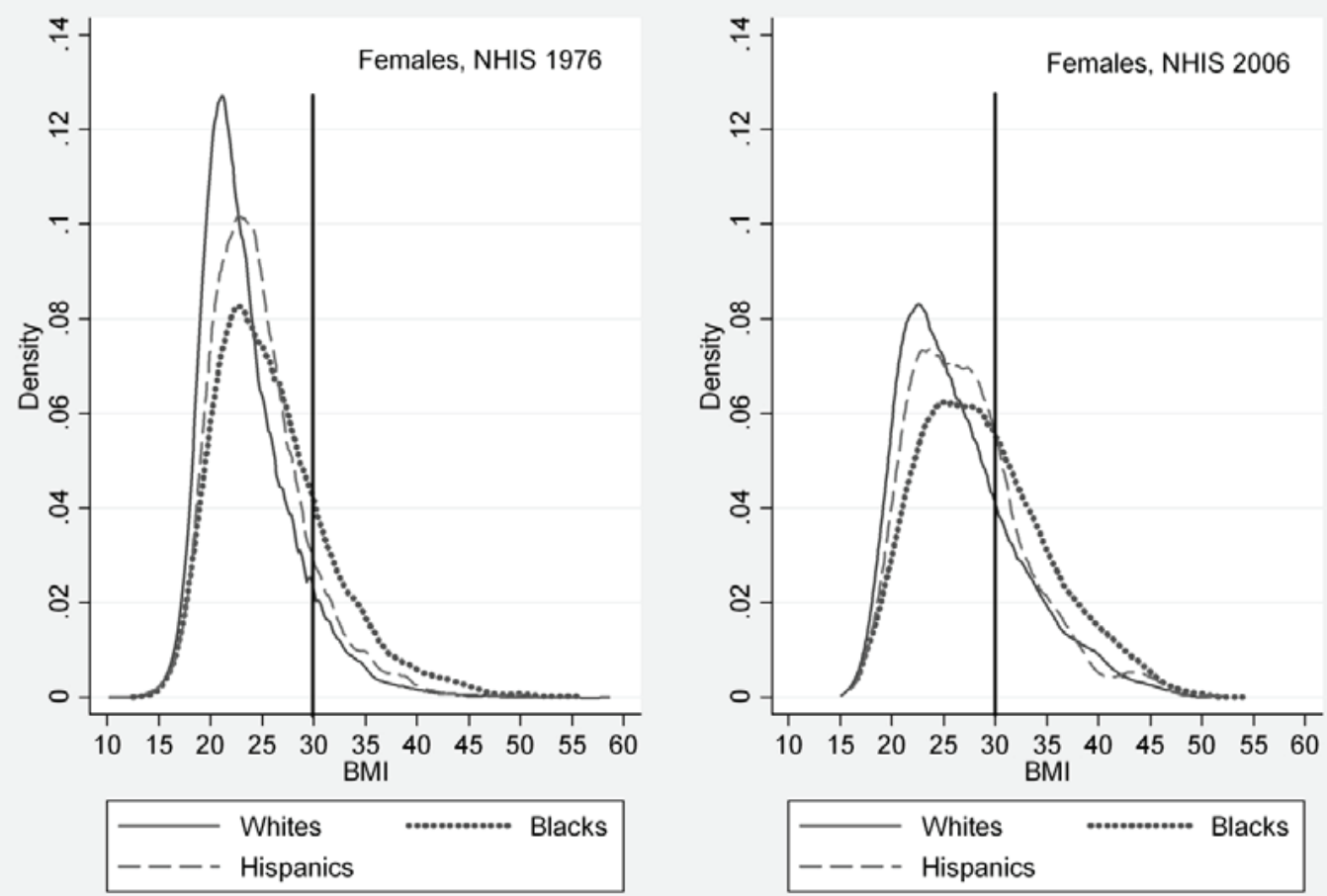

b

Fig. 2. BMI Histogram for a males and $\mathbf{b}$ females in the NHIS. Age 20-74 years. Weighted. The vertical line represents obesity (BMI $\left.\geq 30 \mathrm{~kg} / \mathrm{m}^{2}\right)$. 
Table 2. Marginal effects of schooling and income on BMI; males, NHIS ${ }^{a}$

\begin{tabular}{|c|c|c|c|c|c|c|c|c|c|c|c|c|}
\hline & \multicolumn{3}{|c|}{ 1976-1979 } & \multicolumn{3}{|c|}{ 1980-1989 } & \multicolumn{3}{|c|}{ 1990-1999 } & \multicolumn{3}{|c|}{ 2000-2006 } \\
\hline & [1] & [2] & [3] & [1] & [2] & [3] & [1] & [2] & [3] & [1] & [2] & [3] \\
\hline \multicolumn{13}{|l|}{ All males } \\
\hline Schooling & -0.087 & & -0.083 & -0.091 & & -0.080 & -0.097 & & -0.087 & -0.081 & & -0.072 \\
\hline Income & & -0.009 & -0.003 & & -0.012 & -0.006 & & -0.010 & -0.005 & & -0.005 & -0.003 \\
\hline $\mathrm{N}$ & 91,195 & 91,195 & 91,195 & 262,292 & 262,292 & 262,292 & 207,452 & 207,452 & 207,452 & 58,539 & 58,539 & 58,539 \\
\hline $\mathrm{R}^{2}$ & 0.050 & 0.046 & 0.051 & 0.053 & 0.050 & 0.054 & 0.040 & 0.037 & 0.040 & 0.034 & 0.033 & 0.035 \\
\hline \multicolumn{13}{|l|}{ White males } \\
\hline Schooling & -0.080 & & -0.075 & -0.093 & & -0.080 & -0.109 & & -0.099 & -0.106 & & -0.095 \\
\hline Income & & -0.008 & -0.003 & & -0.012 & -0.007 & & -0.009 & -0.005 & & -0.006 & -0.003 \\
\hline $\mathrm{N}$ & 79,061 & 79,061 & 79,061 & 219,392 & 219,392 & 219,392 & 160,953 & 160,953 & 160,953 & 40,621 & 40,621 & 40,621 \\
\hline $\mathrm{R}^{2}$ & 0.049 & 0.046 & 0.049 & 0.053 & 0.051 & 0.054 & 0.044 & 0.041 & 0.044 & 0.042 & 0.039 & 0.042 \\
\hline \multicolumn{13}{|l|}{ Black males } \\
\hline Schooling & -0.049 & & -0.055 & -0.039 & & -0.047 & -0.007 & & -0.024 & -0.030 & & -0.053 \\
\hline Income & & 0.000 & 0.005 & & 0.001 & 0.005 & & 0.006 & 0.008 & & 0.005 & 0.007 \\
\hline $\mathrm{N}$ & 7,353 & 7,353 & 7,353 & 26,003 & 26,003 & 26,003 & 22,538 & 22,538 & 22,538 & 7,419 & 7,419 & 7,419 \\
\hline $\mathrm{R}^{2}$ & 0.054 & 0.053 & 0.055 & 0.051 & 0.050 & 0.051 & 0.021 & 0.022 & 0.022 & 0.018 & 0.019 & 0.019 \\
\hline \multicolumn{13}{|l|}{ Hispanic males } \\
\hline Schooling & -0.115 & & -0.118 & -0.087 & & -0.075 & -0.046 & & -0.033 & 0.033 & & 0.031 \\
\hline Income & & -0.012 & 0.002 & & -0.017 & -0.009 & & -0.011 & -0.008 & & 0.003 & 0.001 \\
\hline $\mathrm{N}$ & 4,781 & 4,781 & 4,781 & 16,897 & 16,897 & 16,897 & 23,961 & 23,961 & 23,961 & 10,499 & 10,499 & 10,499 \\
\hline $\mathrm{R}^{2}$ & 0.066 & 0.056 & 0.066 & 0.060 & 0.057 & 0.061 & 0.043 & 0.043 & 0.043 & 0.033 & 0.033 & 0.033 \\
\hline
\end{tabular}

aSchooling measured in years. Income measured in thousands of constant USD 2,000.-. Cells report the coefficients of schooling and real personal income from pooled regressions of BMI on schooling (column [1]), real personal income (column [2]), or both (column [3]). Covariates include age, age squared, age by year, age squared by year, and year fixed effects. All regressions use the NHIS sample weights; standard errors have been clustered by PSU. Italic entries are statistically significant at $5 \%$.

similar shifts. Black females (0.024/0.042; $\mathrm{p}=0.0129)$ display an opposite shift; their distribution became a little less right-skewed. This is also indicated for Hispanic females, but the differences are not statistically significant $(0.026 / 0.033 ; \mathrm{p}=0.31)$.

\section{Self-Reported versus Measured BMI}

It is well known that BMI variables based on self-reported height and weight and those based on measurements are usually different [11]. Figure 3a-d presents self-reported (NHANES, NHIS, BRFSS) and measured (NHANES) height and weight over the entire sample period to examine differences across datasets . Results suggest that, in all three datasets, both genders overreport height (with men showing greater bias) and females consistently underreport their weight. The results in the NHIS corroborate previous studies in the NHANES and BRFSS $[27,28]$.

Translating differences in height and weight to differences in BMI, several things can be noted: i) Using the BRFSS or the NHIS, self-report bias is about twice as large for females as for men (an average of two BMI units compared to one; see fig. 1a-c). ii) Survey design seems to matter: the bias is greater in the telephone-based BRFSS compared to the NHANES [27], 
Table 3. Marginal effects of schooling and income on BMI; females, NHIS

\begin{tabular}{|c|c|c|c|c|c|c|c|c|c|c|c|c|}
\hline & \multicolumn{3}{|c|}{ 1976-1979 } & \multicolumn{3}{|c|}{ 1980-1989 } & \multicolumn{3}{|c|}{ 1990-1999 } & \multicolumn{3}{|c|}{$2000-2006$} \\
\hline & [1] & [2] & {$[3]$} & [1] & [2] & [3] & [1] & [2] & [3] & [1] & [2] & [3] \\
\hline \multicolumn{13}{|c|}{ All females } \\
\hline Schooling & -0.319 & & -0.249 & -0.322 & & -0.232 & -0.337 & & -0.242 & -0.312 & & -0.223 \\
\hline Income & & -0.065 & -0.042 & & -0.067 & -0.048 & & -0.055 & -0.039 & & -0.037 & -0.026 \\
\hline $\mathrm{N}$ & 102,707 & 7102,707 & 102,707 & 294,069 & 9294,069 & 294,069 & 232,135 & 5232,135 & 232,135 & 67,662 & 67,662 & 67,662 \\
\hline $\mathrm{R}^{2}$ & 0.096 & 0.086 & 0.106 & 0.085 & 0.084 & 0.098 & 0.061 & 0.061 & 0.072 & 0.050 & 0.050 & 0.059 \\
\hline \multicolumn{13}{|c|}{ White females } \\
\hline Schooling & -0.272 & & -0.221 & -0.281 & & -0.212 & -0.299 & & -0.227 & -0.308 & & -0.237 \\
\hline Income & & -0.049 & -0.032 & & -0.053 & -0.038 & & -0.042 & -0.029 & & -0.029 & -0.020 \\
\hline $\mathrm{N}$ & 86,883 & 86,883 & 86,883 & 237,713 & 3237,713 & 237,713 & 172,212 & 2172,212 & 172,212 & 44,574 & 44,574 & 44,574 \\
\hline $\mathrm{R}^{2}$ & 0.088 & 0.079 & 0.095 & 0.081 & 0.079 & 0.091 & 0.060 & 0.058 & 0.067 & 0.053 & 0.050 & 0.059 \\
\hline \multicolumn{13}{|c|}{ Black females } \\
\hline Schooling & -0.322 & & -0.235 & -0.307 & & -0.221 & -0.321 & & -0.227 & -0.233 & & -0.142 \\
\hline Income & & -0.088 & -0.060 & & -0.078 & -0.054 & & -0.061 & -0.043 & & -0.035 & -0.028 \\
\hline $\mathrm{N}$ & 10,285 & 10,285 & 10,285 & 36,996 & 36,996 & 36,996 & 32,531 & 32,531 & 32,531 & 10,888 & 10,888 & 10,888 \\
\hline $\mathrm{R}^{2}$ & 0.123 & 0.121 & 0.132 & 0.099 & 0.099 & 0.107 & 0.059 & 0.059 & 0.066 & 0.042 & 0.045 & 0.048 \\
\hline \multicolumn{13}{|c|}{ Hispanic females } \\
\hline Schooling & -0.262 & & -0.197 & -0.263 & & -0.182 & -0.247 & & -0.158 & -0.156 & & -0.088 \\
\hline Income & & -0.087 & -0.058 & & -0.088 & -0.062 & & -0.073 & -0.054 & & -0.041 & -0.034 \\
\hline $\mathrm{N}$ & 5,539 & 5,539 & 5,539 & 19,360 & 19,360 & 19,360 & 27,392 & 27,392 & 27,392 & 12,200 & 12,200 & 12,200 \\
\hline $\mathrm{R}^{2}$ & 0.106 & 0.101 & 0.117 & 0.093 & 0.094 & 0.106 & 0.066 & 0.071 & 0.079 & 0.047 & 0.054 & 0.056 \\
\hline
\end{tabular}

aSchooling measured in years. Income measured in thousands of constant USD 2,000.-. Cells report the coefficients of schooling and real personal income from pooled regressions of BMI on schooling (column [1]), real personal income (column [2]), or both (column [3]). Covariates include age, age squared, age by year, age squared by year, and year fixed effects. All regressions use the NHIS sample weights; standard errors have been clustered by PSU. Italic entries are statistically significant at $5 \%$.

but there is also a difference between the NHIS and the NHANES self-reports, both of which are based on in-person interviews. iii) Shifts in the BMI distribution over time are similar, regardless of whether self-reports or measurements are used, and underreporting of BMI increases with BMI $[28,29]$. iv) Although the use of self-reports underestimates the prevalence of obesity, time trends are consistent between measured and self-reported data.

\section{The SES-BMI Gradient}

A concise way of examining the relationship between BMI and SES is to look at gradients (marginal effects). Table 2 and table 3 answer the following questions: i) How do these gradients differ by race/ethnicity and gender? ii) Have these gradients changed over time? iii) Given that both education and income have a positive effect on health, how much of the education effect can be accounted for through changes in income level (e.g., more education being associated with a higher income), and how much of the income effect can be accounted for through changes in education level? Estimates are presented using data from the NHIS. Results for all males and all females (combined across race/ethnicity groups) in the BRFSS 

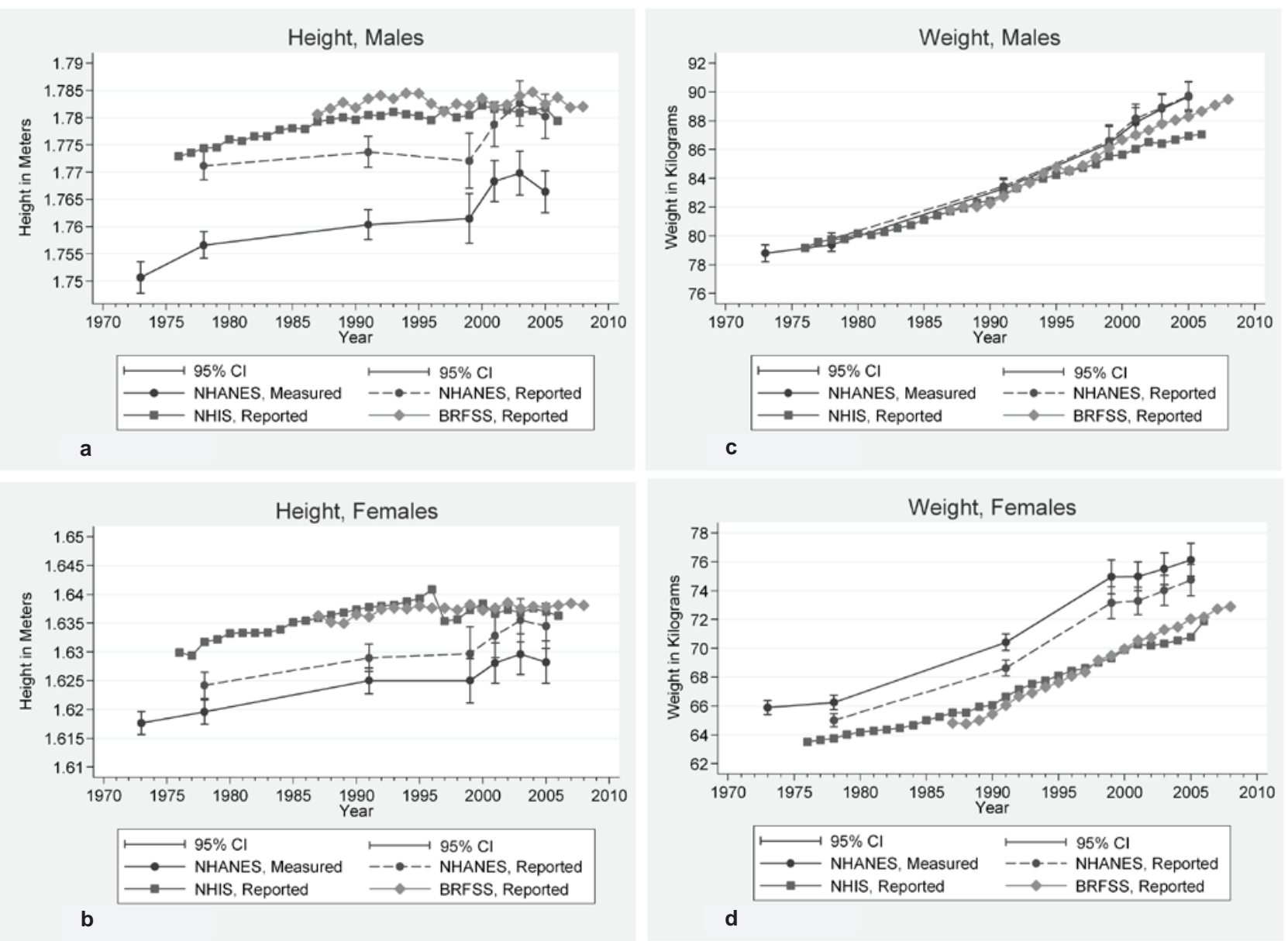

Fig. 3. Comparison of means across datasets and self-reports versus measurements. a height, males; b height, females; c weight, males; d weight, females. Age 20-74 years. Weighted and age-adjusted to the 2000 Census.

and NHANES are very similar and available in the online supplementary material (Appendix table 1 and 2, available at $h t t p: / / c o n t e n t . k a r g e r . c o m / P r o d u k t e D B / p r o d u k t e . a s p ? d o i=337018$ ).

Regarding the first question, an extra year of schooling is associated with a modest decrease in BMI for men (about 0.08 units) and a sizeable decrease for women (0.25-0.3 units). Schooling gradients for Black men are about half as large as those for Whites. These differences are consistent with lower wage returns to education for Blacks, possibly caused by lower-quality schooling [30]. There are no such differences among females, although the effects for Hispanic females are always somewhat lower than those for Whites and Blacks. Looking at the effect of income, an extra USD 1,000.- in real personal income is associated with a modest decrease in BMI of about 0.005-0.016 units for men and 0.04-0.07 units for women. Among males, only Whites show a consistently inverse relationship. For females the effects are similar across race/ethnicity groups, with Hispanics displaying the strongest effects.

The marginal effects of schooling and income are not easily comparable due to their different scales. One way to address this is to compare the effects of a one-standard-deviation increase in the respective variables. Using the pooled 1976-2006 NHIS dataset, a onestandard-deviation increase in schooling is associated with a change in BMI of -0.25 (White 
males), -0.12 (Black males), and -0.17 units (Hispanic males), while a one-standard-deviation increase in real personal income is associated with a change in BMI of -0.09 (White males), 0.11 (Black males), and -0.05 units (Hispanic males). For women, these estimates are $-0.60,-0.64$, and -0.59 (schooling), and $-0.49,-0.56$, and -0.62 (real personal income). This highlights two observations: i) In this population, education and income have associations with BMI of similar magnitude among women, while among men education has a bigger impact; ii) the relationships for women are 2.5-12 times stronger than for men.

The second question concerns changes in the gradients over time. Previous studies have found increasing education gradients in mortality and other health outcomes over time, which may contribute to the observed increase in health inequalities [30]. The author is not aware of any results relating to BMI. For White males and, to a lesser extent, White females, coefficient estimates in the NHIS suggest that schooling gradients have increased in absolute value over time, but the differences are not statistically significant. For Black males and females the gradients have stayed roughly constant. Among both Hispanic males and females schooling gradients have decreased in absolute value (indeed, for Hispanic men, they were slightly positive in 2000-2006), possibly due to the increases in immigration of low-educated Hispanics over that time period. There are no clear trends in the income-BMI gradients among men. Among women of all race/ethnicity groups the gradients have decreased a bit in absolute value.

With regard to the last question - the relative importance of education and income - the evidence suggests that, for males, income accounts for only a small part of the education effect, while education accounts for a significant share of the income effect. According to table 2 and table 3, including income in a regression of BMI on schooling reduces the schooling coefficient by $10 \%$ for males, while the opposite reduces the income coefficient by $50 \%$. For females these effects are similar at around $30 \%$.

\section{Discussion}

To the author's knowledge, this study is the first to estimate marginal effects of SES on BMI across various gender and race/ethnicity groups. Marginal effects summarize the SES-BMI gradient in a single number, facilitating comparisons across groups and over time. Previous studies have extensively documented a strong inverse relationship between SES and obesity for women while for men the relationship is much weaker. This has been observed in this study as well, for all race/ethnicity groups and datasets. However, the effects of education and schooling are distinct and vary by gender. For men, a one-standarddeviation increase in education (about 3 years of schooling) is associated with a reduction in BMI of about 0.2 units, while a one-standard-deviation increase in real personal income (about USD 16,000.-) is correlated with a 0.07 unit decrease in BMI (for Black men, more income is associated with an increase in BMI of 0.11 units). For women, on the other hand, such increases are associated with declines in BMI of about 0.5-0.6 units. This suggests that: i) In this population, education and income have similar effects for women while for men education is stronger; ii) effects are considerably stronger for women than for men.

Previous work in the NHIS [30] found that income, health insurance coverage, and other controls together account for about one third of the total effect of education on BMI in White males. A related study [31], also examining White males, reported a 16\% decrease in the education coefficient for BMI in the NHIS and 13\% in the Health and Retirement Study when income was included. The current study provides more detail on this relationship. Among males, income accounts for $10 \%$ of the total effect of education on BMI; for females, the effect is about $30 \%$. These estimates are also similar across the three datasets. 
Earlier studies of the NHANES and BRFSS have shown that the growth in extreme obesity is due to certain changes in the BMI distribution which has both shifted to the right and become more right-skewed over time $[3,10]$. Such shifts can also be found in the NHIS long-run perspective from 1976 to 2006. It is strongest for White and Black men as well as White females. The distribution for Black females shifted strongly to the right as well, but became less skewed. Hispanic females experienced an even shift. Overall, this casts Hispanics as either relatively similar to Whites and Blacks (men), or somewhere in between these other two groups (women), in terms of overall prevalence and distribution shifts.

The other question this study investigated concerns similarities and differences across three big national health datasets - the NHANES, NHIS, and BRFSS. Differences across surveys in BMI prevalence and time trends could be due to various reasons, such as measurement bias, sampling bias, or non-response bias [32]. The specific questions that were asked in the surveys to elicit self-reports of height and weight are virtually identical (online supplementary material, available at http://content.karger.com/ProdukteDB/produkte. asp?doi=337018). The exam-based measurements in the NHANES were taken by trained technicians following standard protocols and as such presumably suffer little from measurement bias. Conversely, there is substantial evidence that height and weight based on self-reports is heavily biased, and this is evident when comparing BMI variables from the NHIS and BRFSS to the exam-based measures. Moreover, while the NHANES and NHIS both use in-person surveys, the BRFSS is conducted via phone. Phone respondents may exhibit more social desirability response bias [33]; all other things equal, BMI measurements in the BRFSS might therefore be lower than those in the NHIS and NHANES.

All three datasets are designed to produce samples representative of the non-institutionalized civilian US population, yet sampling bias could arise in various ways. The NHANES, for instance, used 65 PSUs in its first series, 64 in the second, 81 in the third, and 15 per year since 1999 [13]. Given the wide variation in obesity rates across states [10], it is possible that sites with higher or lower obesity prevalence were over-represented in any given series [32]. The BRFSS, on the other hand, being based on phone interviews, is subject to wide and systematic variations in landline phone coverage and has only recently started to sample households with cell phones but no landline. The NHIS uses in-person interviews and selects a much higher number of PSUs (up to 428, time-varying [15]), but suffers from re-designs every 10-15 years that might affect comparability over time.

There might also be biases due to non-response, depending on both the response rates and the differences between responders and non-responders. In the NHANES, interview response rates have decreased from over $90 \%$ to about $80 \%$ while response rates for the medical examinations have been fairly constant around 75-80\% [13]. Response rates in the BRFSS averaged 65-70\% from inception to about 1996 and have since dropped to around $50 \%$ [34]. Non-response rates in the NHIS have increased from around $4 \%$ in the early $1990 \mathrm{~s}$ to more than $12 \%$ by 2005 [35]. Lower response rates are worrisome on their own, but they only affect non-response bias to the extent that there are significant differences between responders and non-responders [32]. To the author's knowledge, no information regarding differences between responders and non-responders is available for any of these three health surveys.

Sampling bias and non-response bias may explain some of the differences in demographics observed across the three surveys. For instance, the BRFSS has a lower percentage of females and Blacks as well as higher average educational attainment and personal income compared to the NHANES and NHIS. Conversely, the NHANES has a higher fraction of people with less than 12 years of schooling than the other two surveys. Since these SES variables are positively related to BMI, it is possible that the NHANES overestimates obesity prevalence while the BRFSS underestimates it [32]. 
And yet, given these differences and potential biases, most BMI-related measures and trends are quite similar across the three surveys (with the exception of underreported prevalence rates in the NHIS and BRFSS, which rely on self-reports). They have similar ranks for at-risk groups and display similar marginal effects. This suggests that the NHIS and BRFSS are quite valid for studying associations between demographics, socioeconomic status and BMI, and are especially valuable for subgroup analysis, where the NHANES lacks statistical power. Note that some issues do require correct prevalence estimates, such as calculating the excess health care costs associated with obesity and overweight.

The potential increase in self-reporting bias due to the correlation between BMI level and self-reporting bias in BMI is an area of concern [27, 29]. More exam-based measures would alleviate this issue; it is encouraging that the BRFSS is launching a pilot project to take physical measurements of part of its sample [36].

Lastly, this study found that measurements based on self-reports available in the NHANES differ not only from those calculated using exam-based variables but also from self-reported measures in the other two datasets (this is also briefly alluded to in $[37,38]$ ). This is a surprising result and warrants further research. Speculatively, the bias usually found in self-reported measurements might have been reduced in the NHANES due to knowledge about the upcoming physical exam. It also suggests that adjusting self-reports in other datasets using the difference between self-reports and measured height and weight in the NHANES is not valid (performed, for instance, in $[39,40]$ ). More generally, exploring differences in self-reported and measured BMI by race/ethnicity and SES would be an interesting topic for future research.

This study is not without limitations. Selecting the most relevant results for presentation necessitated the exclusion of other findings (e.g. age stratification of BMI trends). The outcome of interest is BMI, which is a flawed measure of obesity [12]; however, it is still widely used and the only measure available in all three datasets analyzed in this study. SES-BMI gradients were derived using OLS; coefficients represent correlations and do not necessarily allow a causal interpretation. The model also assumes a linear relationship between SES and BMI. The results on SES-BMI gradients are derived for a US population and further research in other populations would be desirable to assess generalizability. Lastly, the study did not examine the sensitivity of results regarding data preparation methods and inclusion/exclusion criteria.

In summary, while there are clear differences in demographics, survey methods, and BMI measurement techniques across the three datasets, their accounts of BMI trends and of associations between SES and BMI are similar. The exact patterns and distributional shifts vary by gender and race/ethnicity. Race/ethnicity differences exist even after accounting for education and income. These disparities should be studied further to identify environmental and/or biological components explaining the differential patterns.

\section{Acknowledgements}

The author gratefully acknowledges the feedback provided by Douglas L. Miller, A. Colin Cameron, J. Paul Leigh, C. Daniel Mullins and Eberechukwu Onukwugha. All errors are the author's.

\section{Disclosure Statement}

No external funding was provided for the conduct of the study or the preparation of the paper. The author has no relevant conflicts of interest. This work reflects the views of the author alone and is not associated with HealthCore, Inc. 


\section{References}

- 1 Flegal KM, Carroll MD, Kuczmarski RJ, Johnson CL: Overweight and obesity in the United States: prevalence and trends, 1960-1994. Int J Obes Relat Metab Disord 1998;22:39-47.

- 2 Flegal KM, Carroll MD, Ogden CL, Curtin LR: Prevalence and trends in obesity among US adults, 1999-2008. JAMA 2010;303:235-241.

3 Ruhm C: Current and future prevalence of obesity and severe obesity in the United States. Forum Health Econom Policy 2007;10:1-26.

- 4 Wang Y, Beydoun MA, Liang L, Caballero B, Kumanyika SK: Will all Americans become overweight or obese? Estimating the progression and cost of the US obesity epidemic. Obesity (Silver Spring) 2008;16:23232330.

5 James WP: The epidemiology of obesity: the size of the problem. J Intern Med 2008;263:336-352.

- 6 Danaei G, Ding EL, Mozaffarian D, Taylor B, Rehm J, Murray CJ, Ezzati M: The preventable causes of death in the United States: comparative risk assessment of dietary, lifestyle, and metabolic risk factors. PLoS Med 2009;6:e1000058.

7 Malnick SD, Knobler H: The medical complications of obesity. QJM 2006;99:565-579.

8 McLaren L: Socioeconomic status and obesity. Epidemiol Rev 2007;29:29-48.

- 9 Sobal J, Stunkard AJ: Socioeconomic status and obesity: a review of the literature. Psychol Bull 1989;105: 260-275.

10 Wang Y, Beydoun MA: The obesity epidemic in the United States-gender, age, socioeconomic, racial/ethnic, and geographic characteristics: a systematic review and meta-regression analysis. Epidemiol Rev 2007;29: 6-28.

11 Gorber SC, Tremblay M, Moher D, Gorber B: A comparison of direct vs. self-report measures for assessing height, weight and body mass index: a systematic review. Obes Rev 2007;8:307-326.

12 Burkhauser RV, Cawley J: Beyond BMI: the value of more accurate measures of fatness and obesity in social science research. J Health Econ 2008;27:519-529.

13 Centers for Disease Control and Prevention: National Health and Nutrition Examination Survey Data. 2010 (cited August 21, 2010); available from www.cdc.gov/nchs/nhanes.htm.

14 Centers for Disease Control and Prevention: National Health Interview Survey. 2010 (cited August 21, 2010); available from www.cdc.gov/nchs/nhis.htm.

15 Minnesota Population Center and State Health Access Data Assistance Center: Integrated Health Interview Series: Version 2.0. 2008 (cited August 21, 2010); available from www.ihis.us/ihis/.

16 Centers for Disease Control and Prevention: Behavioral Risk Factor Surveillance System Survey Data. 2010 (cited August 21, 2010); available from www.cdc.gov/brfss/index.htm.

17 Centers for Disease Control and Prevention: 2009 BRFSS Overview. 2009 (cited August 21, 2010); available from www.cdc.gov/brfss/technical_infodata/surveydata/2009/overview_09.rtf.

18 Centers for Disease Control and Prevention: About BMI for Adults. 2010 (cited August 21, 2010); available from www.cdc.gov/healthyweight/assessing/bmi/adult_bmi/index.html.

19 Klein R, Schoenborn C: Age adjustment using the 2000 projected U.S. population. Healthy People 2000 Stat Notes 2001;20:1-9.

20 StataCorp: Stata Statistical Software: Release 10. College Station, StataCorp LP, 2007.

21 Truong KD, Sturm R: Weight gain trends across sociodemographic groups in the United States. Am J Public Health 2005;95:1602-1606.

-22 Nelson DE, Powell-Griner E, Town M, Kovar MG: A comparison of national estimates from the National Health Interview Survey and the Behavioral Risk Factor Surveillance System. Am J Public Health 2003;93: 1335-1341.

23 Beydoun MA, Wang Y: Gender-ethnic disparity in BMI and waist circumference distribution shifts in US adults. Obesity (Silver Spring) 2009;17:169-176.

-24 Flegal KM, Troiano RP: Changes in the distribution of body mass index of adults and children in the US population. Int J Obes Relat Metab Disord 2000;24:807-818.

25 Freedman DS, Khan LK, Serdula MK, Galuska DA, Dietz WH: Trends and correlates of class 3 obesity in the United States from 1990 through 2000. JAMA 2002;288:1758-1761.

-26 Sturm R: Increases in clinically severe obesity in the United States, 1986-2000. Arch Intern Med 2003;163: 2146-2148.

27 Ezzati M, Martin H, Skjold S, Vander Hoorn S, Murray CJ: Trends in national and state-level obesity in the USA after correction for self-report bias: analysis of health surveys. J R Soc Med 2006;99:250-257.

28 Merrill RM, Richardson JS: Validity of self-reported height, weight, and body mass index: findings from the National Health and Nutrition Examination Survey, 2001-2006. Prev Chronic Dis 2009;6:A121.

-29 Villanueva EV: The validity of self-reported weight in US adults: a population based cross-sectional study. BMC Public Health 2001;1:11.

30 Cutler D, Lleras-Muney A: Education and Health: Evaluating Theories and Evidence. NBER Working Paper No. 12352, 2006.

-31 Cutler DM, Lleras-Muney A: Understanding differences in health behaviors by education. J Health Econ 2010;29:1-28. 
-32 Yun S, Zhu BP, Black W, Brownson RC: A comparison of national estimates of obesity prevalence from the Behavioral Risk Factor Surveillance System and the National Health and Nutrition Examination Survey. Int J Obes (Lond) 2006;30:164-170.

-33 Holbrook AL, Green MC, Krosnick JA: Telephone versus face-to-face interviewing of national probability samples with long questionnaires - comparisons of respondent satisficing and social desirability response bias. Public Opinion Q 2003;67:79-125.

34 Johnson D: Addressing the Growing Problem of Survey Nonresponse. 2010. Mimeo. Available from $w w w$. ssri.psu.edu/survey/Nonresponse1.ppt.

35 Stussman B, Simile C: Data Quality of the NHIS and the Contact History Instrument. 2006. Mimeo. Available from www.cdc.gov/nchs/ppt/duc2006/strussman_13.ppt.

36 Centers for Disease Control and Prevention: Improvements to BRFSS Methodology, Design, and Implementation. 2010 (cited August 21, 2010); available from www.cdc.gov/BRFSS/pubs/methodology.htm.

37 Reither EN, Utz RL: A procedure to correct proxy-reported weight in the National Health Interview Survey, 1976-2002. Popul Health Metr 2009;7:2.

-38 Stommel M, Schoenborn CA: Accuracy and usefulness of BMI measures based on self-reported weight and height: findings from the NHANES \& NHIS 2001-2006. BMC Public Health 2009; 9:421.

39 Cawley J: The impact of obesity on wages. J Hum Resour 2004;39:451-474.

40 Chou SY, Grossman M, Saffer H: An economic analysis of adult obesity: results from the Behavioral Risk Factor Surveillance System. J Health Econ 2004;23:565-587. 